Acta Technologica Agriculturae 1

Nitra, Slovaca Universitas Agriculturae Nitriae, 2021, pp. 48-54

\title{
ARTIFICIAL INTELLIGENCE-DRIVEN AUTONOMOUS ROBOT FOR PRECISION AGRICULTURE
}

\author{
Ivan BELOEV, Diyana KINANEVA, Georgi GEORGIEV, Georgi HRISTOV*, Plamen ZAHARIEV \\ University of Ruse "Angel Kanchev", Ruse, Bulgaria
}

\begin{abstract}
In the recent years, robotic systems became more advanced and more accessible. This has led to their slow, but stable integration and use in different processes and applications, including in the agricultural domain. Nowadays, agricultural robots are developed with the aim to replace the human labour in the otherwise exhausting, time-consuming or dangerous activities. Agricultural robotic systems provide many advantages, which can differ based on the type of the robot and its sensors, actuators and communication systems. This paper presents the design, the construction process, the main characteristics and the evaluation of a prototype of a small-scale agricultural robot that can be used for some of the simplest activities in agricultural enterprises. The robot is designed as an end-user autonomous mobile system, which is capable of self-localization and can map or inspect a specific farming area. The decision-making capabilities of the robot are based on artificial intelligence (Al) algorithms, which allow it to perform specific actions in accordance to the situation and the surrounding environment. The presented prototype is in its early development and evaluation stages and the paper concludes with discussions on the possible further improvements of the platform.
\end{abstract}

Keywords: computer vision algorithms; robotics; simultaneous localization and mapping; visual navigation; visual odometry; smart farming

Precision agriculture, and more specifically precision farming, involves the use of modern information and communication technologies, which are implemented with the aim to increase crop yields and profitability. At the same time, precision agriculture leads to an overall decrease of the amount of investments and resources, in terms of arable land, water, fertilizers, herbicides, insecticides, etc., which are needed to grow the crops. Some of the technologies that are used in the precision agriculture domain, include online weather forecasts, sensor systems and networks, satellite systems, NDVI and photographic systems, systems for weed detection, etc. The information provided by these solutions needs to be analysed before it can produce agronomic recommendations or instructions, but in this way precision agriculture can provide farmers with the knowledge about the needed resources, as well as with information in what amount and when they should be applied.

The timely delivery of the agronomical recommendations is a key factor for the improvement of the quality and the quantity of yields. However, these recommendations typically involve or require a specific action to take place. Of course, farmers do not have to perform these activities by hands or on their own - they can use robots and mobile autonomous systems (Krastev and Georgiev, 2015). The use of robots for precision agriculture is not a new trend. Over several decades, the robots for precision agriculture have been subject to developments and upgrades and their modern versions are smarter and can make decisions on their own by using different input parameters (Amer et al., 2015).
There are several different types of precision agriculture robots (Cheein and Carelli, 2013). The ground-based open space robots are probably the most widely distributed and used (Cosgrove, 2017). The robots for greenhouse or indoor farming form the second group. The possibility to monitor the crops from the air has provided priceless information to farmers in the past and has increased the yields significantly (Yaghoubi et al., 2013). For this reason, the aerial unmanned robotic platforms have slowly defined a standalone category of precision agriculture robots. In the last decade, the main research efforts in the precision agriculture area are focused on the development of small-scale flexible and highly mobile solutions, which can be used both indoors and outdoors, and even in urban conditions, like for example on the rooftops of the buildings (Farmaid, 2020) or in backyards.

The improvement of the autonomous movement and navigation capabilities of precision agriculture robots is one of the most widely researched areas (Bakker et al., 2010). The most trivial navigation method is based on satellite triangulation and allows the robot to move through the open field towards the desired endpoint (Bakker et al., 2010). Due to numerous disadvantages of this approach and its inaccuracy, many of the modern agricultural machines (Claas 700, 2020; Fendt, 2020; John Deere, 2020) have also visual feedback control - using optical or electro-optical cameras (Pierpaoli et al., 2013), ultrasonic proximity sensors or Light Imaging, Detection and Ranging (LIDAR) systems. The movement of the robots in the field is also a significant challenge for the ground-based robots

Contact address: Georgi Hristov, University of Ruse "Angel Kanchev", Department of Telecommunications, Ruse, Bulgaria, e-mail: ghristov@uni-ruse.bg 
(Hernández et al., 2013), as well as the improvement of the tools used for seeding, transplanting, fertilizing and harvesting (Claas Isaria, 2020). The indoor robots also suffer from many disadvantages and are usually limited to the manipulation on just one fruit or vegetable, mainly due to the limitations of the technological solutions and the restricted working spaces (Belforte et al., 2006, Farmaid, 2020). On the other hand, Unmanned Aerial Vehicles (UAVs), like the T16 by DI I I Agras T16, 2020), can be used for insect and weed control for a variety of crops, such as rice, wheat, corn, citrus trees, cotton, and much more. Nevertheless, these robotic systems are very difficult to control and sometimes require special permits to be operated (Hristov et al., 2016).

In this paper we present our efforts to develop a small-scale mobile robot for precision agriculture that can be used both indoors and outdoors. Our aim is focused on the evaluation of modern technologies that can be used

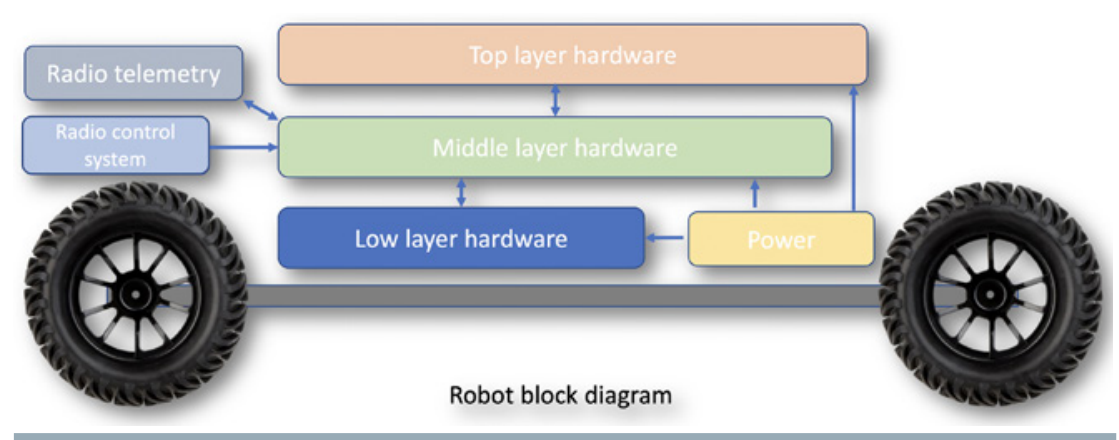

Fig. 1 Block diagram of the general architecture of the agricultural robot prototype

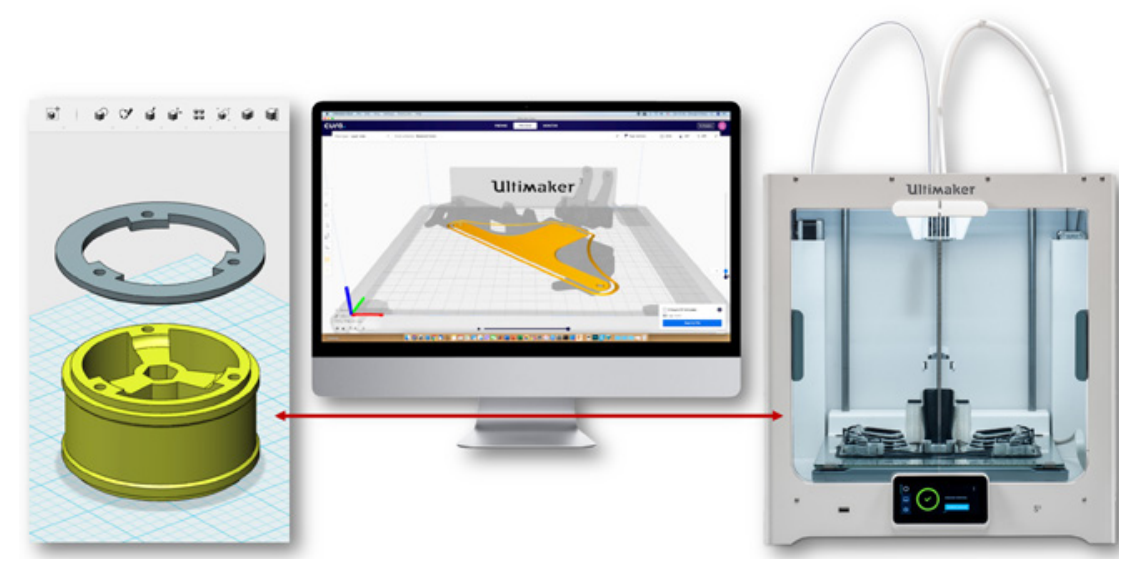

Fig. 2 Design of the different elements of the robot chassis, adjustment of the components, positioning and preparation of the models in the software for $3 \mathrm{D}$ printing for the construction of the robot, and we are driven by the goal to produce the cheapest possible solution, which to perform the basic tasks, like weed detection, spraying, etc. To solve the navigation issues described above, we have investigated the possibility to use both GPS-based navigation solution and a visual navigation solution that is based on depth and tracking cameras.

\section{Material and methods}

The presented robot prototype is a highly efficient autonomous smallscale, ground-based mobile platform that uses advanced computer vision methods to distinguish different objects, colours and plants and can apply a liquid mixture using its on-board sprinklers. The general architecture of the robot, its hardware layers and the connections between them are presented in Fig. 1. will be easy to operate and will be able 


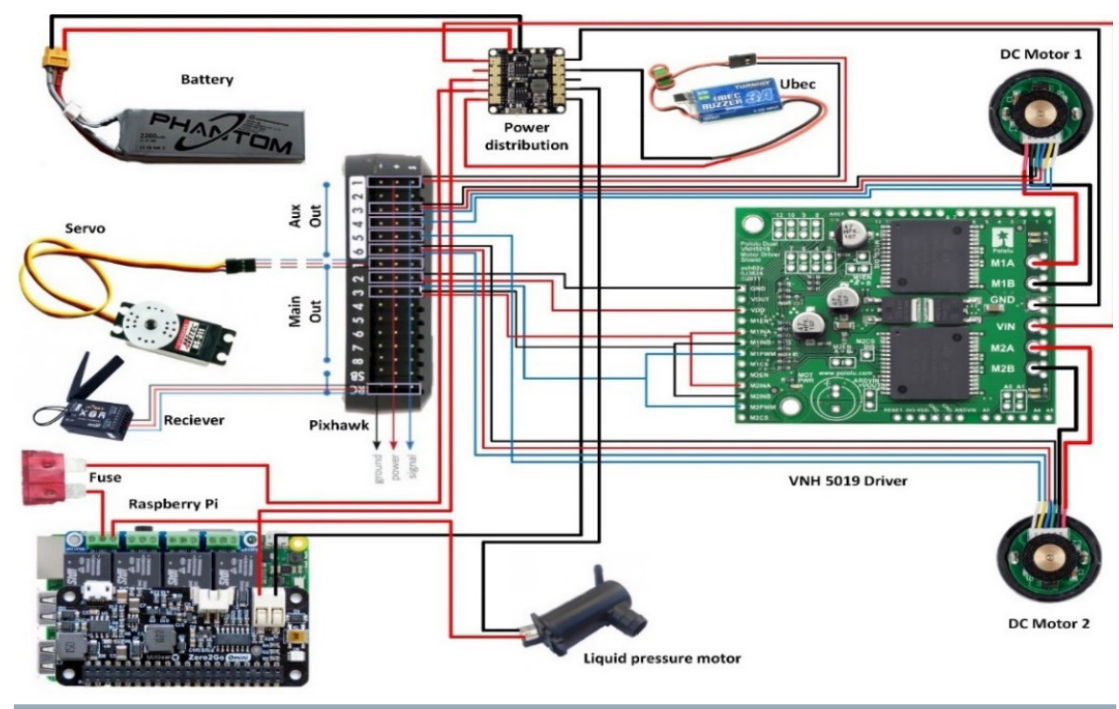

Fig. 3 Wiring and power supply diagram of the smart agricultural robot

purpose of the robot, as well as for remotely controlled drones and cars.

Figure 3 presents the wiring and power supply diagram of the robot. Additionally, the figure shows how the DC motors are connected to the driver board and how the connection between the board and the Pixhawk microcontroller is made. The drivers are installed in a mirror configuration, so it was required to reverse the polarity of the power supply to one of the motors. Since the motors are $D C$, the change of the polarity will reverse the direction of rotation of the motor shaft and will synchronize the direction of rotation of both motors. The Pixhawk autopilot controls the motor driver via PWM signals. These signals have different levels, in order to determine the direction of the movement. A high-level signal will drive the robot forward, while a low-level signal will drive the robot backwards. The Pixhawk microcontroller controls all the hardware components of the middle layer, which are above the DC motors, the motor driver shield and the servo controller. Some of the connected components are required, while others are optional. For example, the primary GPS and compass are required to provide the autopilot with positioning data during movement, while the secondary GPS system is an optional component and is used to improve the localization precision.

The precision agriculture robotic platform that is presented in this paper relies on computer vision and image processing algorithms for the automatic detection of weeds and colours, as well as for the visual navigation and the avoidance of obstacles. Supervised machine learning approaches, which are relevant to the functionality of the robot, were investigated and used for the evaluation of its performance. They include four machine learning problems, namely classification, localization, object detection and segmentation, which are somewhat common, but differ in the produced outputs (Fig. 4).

In order to perform the object detection tasks, we use deep neural classification models. Their convolutional layers are the key component, which enables image processing and object detection. Usually, they are referred to as filters, which extract features from the previous layers in order to transform the input data in a form that is more useful for representation.

The workflow for the training of the robot to recognize weeds in a field with agricultural crops is presented in Fig. 5. The TensorFlow object detection API (Huang, 2017) is used as training platform. The workflow contains four main stages preparation of the environment, preparation of the datasets, training and then implementation of the object detection model (Fig. 5).

The input dataset is required to contain images that have objects of interest - for the agricultural robot the images must contain different kind of weeds that are often found in the field. To train a good detection model, TensorFlow requires hundreds of thousands of images. However, instead of training a model from scratch, it is possible to retrain a pre-trained neural network model and then use smaller amounts of images. We have used more than 300 photographs of different
This is Tomato!

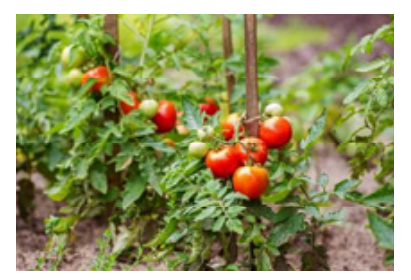

Classification
This is Tomato!
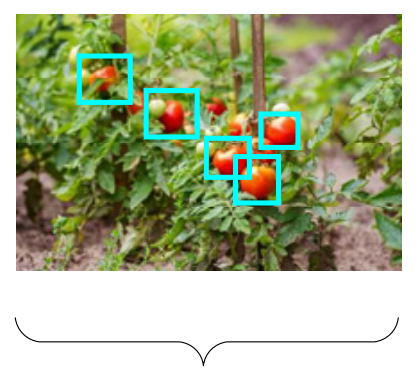

Classification

and localization
This is Tomato, Leaf, Wood!
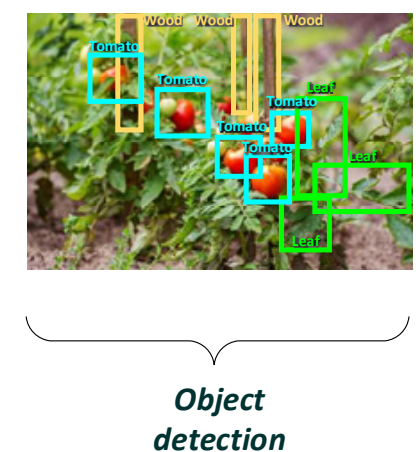

This is Tomato, Leaf, Wood!
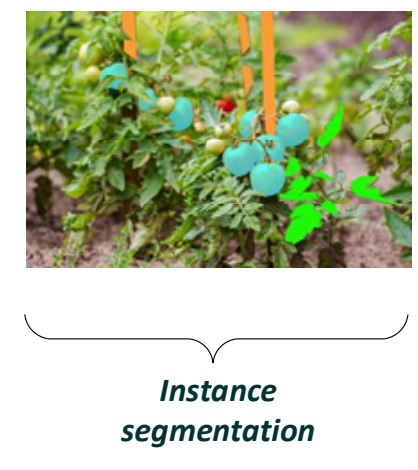

Fig. 4 Comparison among the classification, localization, object detection and segmentation problems 


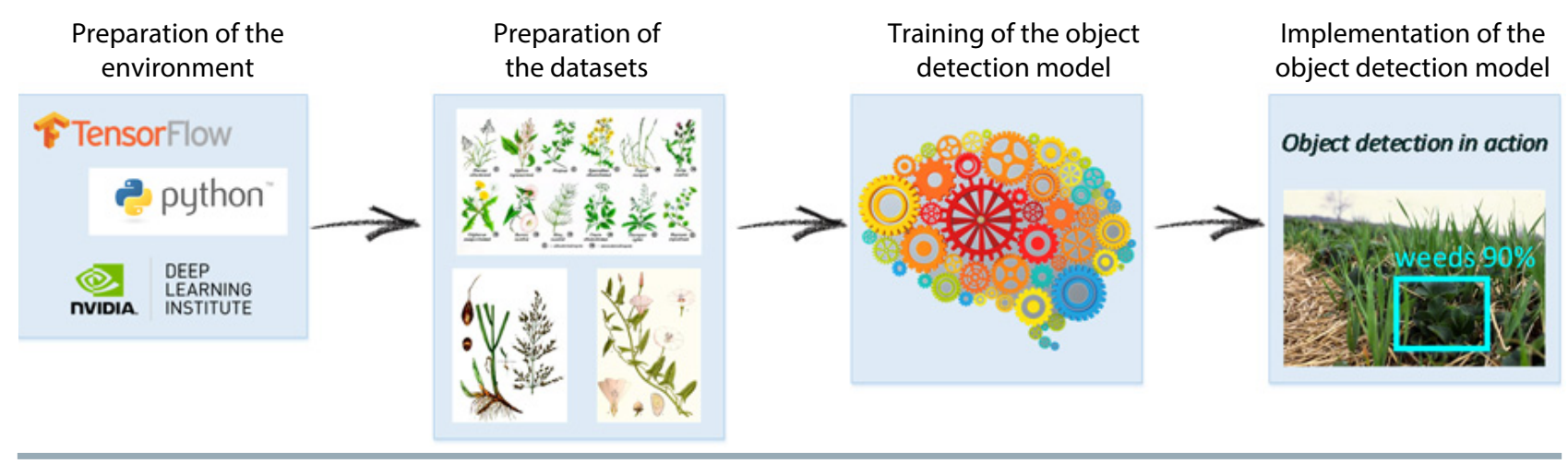

Fig. 5 Workflow for development of a computer vision solution for weed detection

weeds for the training of our solution. Twenty percent of the images were set aside for testing and to avoid overfitting the model, while the rest were used for the training process, like in Abbaspour-Gilandeh (2019). After collecting the desired number of images, they were labelled to show the classifier where the object of interest resides in the image. As a training model, the Faster R-CNN with Inception version 2 was used. It is available online in the TensorFlow model zoo (TensorFlow object detection zoo, 2017). The Faster R-CNN is canonical model for deep-learning object detection. The time it takes to train the model depends on the number of images, their resolution and the hardware parameters of the computing unit. After the training, the last step in the process is to export the trained model and use it in practice.

In specific situations, the robot might have to implement autonomous tasks that are more complex. To do this, the robot can be configured to navigate on its own and without any predefined routes, but again without damaging the crops. In this case, the goal of the robot is to construct a map of the unknown environment and to localize itself on it. In the computer sciences, this problem is known as the Simultaneous Localization and Mapping (SLAM) problem. It is also considered to be fundamental for the robotics domain and the solution to it allows the robots to become truly autonomous (Bailey and Durrant-Whyte, 2006). SLAM actually combines two problems - the localization problem and the mapping problem, which are easy to solve on their own. Nowadays, it is somewhat simple to create a map that is based on a known position, which is the mapping problem, and it is also relatively easy to obtain your own location within the known map - the localization problem. However, if both problems exist at the same time and if they have to be solved simultaneously, their combination proves to be challenging and complex. Fortunately, there are several known algorithms that can solve the SLAM problem, so the research efforts in this area are concentrated on their improvement in terms of computational efficiency, as well as on the provisioning of consistent and accurate estimate about the map and the position of the robot (Durrant-Whyte and Bailey, 2006).

The most recent scientific efforts to solve the SLAM problem are based on visual methods for positioning, also known as visual odometry. This approach allows the estimation of the location of the robot based on the 3D motion of the camera in space. There are two main ways to implement a visual odometry solution - by single camera (monocular) or by two cameras (stereoscopic). The later calculates the depth by estimating the disparities between the matching key points in the images from both cameras. The visual odometry is a robust technique for autonomous navigation, motion tracking and obstacle detection and avoidance (Cviklovič et al., 2016). It allows a robot to localize itself by using only a stream of images captured by the camera attached to it. For this reason, we have decided to proceed with the implementation of this solution for the developed platform.

\section{Results and discussion}

For the implementation of the computer vision tasks by the developed small-scale agricultural robot, we have used the widely available Raspberry Pi 3 Model B+ microcomputer (Raspberry 3, 2020). It is the core of the top-level hardware layer of the robot (Fig. 1). This specific version of the Raspberry Pi supports several cameras, including the commercially available Raspberry Pi cameras and the more advanced Intel Real Sense Depth and Tracking Cameras.

To detect different colours with the robot, we have developed a custom Python script that allows the detection of a specific predefined colour. For the purpose of the experiment, we have assumed that the red colour is representing the presence of weeds. After their detection, the robot will treat the weeds locally by spraying them with a liquid solution. The running of the programming script requires a pre-installed version of OpenCV. The code that is used for the tests of the computer vision algorithm is expected to recognise only colours. The process of colour recognition requires the conversion of the colour diagram of the images from the camera from RGB (red-green-blue) colour format to HSV (hue-saturation-value) format. The need to convert the colour formats comes from the fact that when describing the RGB colours, the colour components of a given object in the image are related to the amount of light that falls on the object. This significantly obstructs the colour distinction of the objects. On the other hand, the HSV colour system is much better for image description, because it uses hue, saturation and value. The computer vision algorithm that is used by the robot in the evaluation scenario is presented in the block diagram below (Fig. 6).

The algorithm is set to recognize two colours - red and green, which are very common for the agricultural 


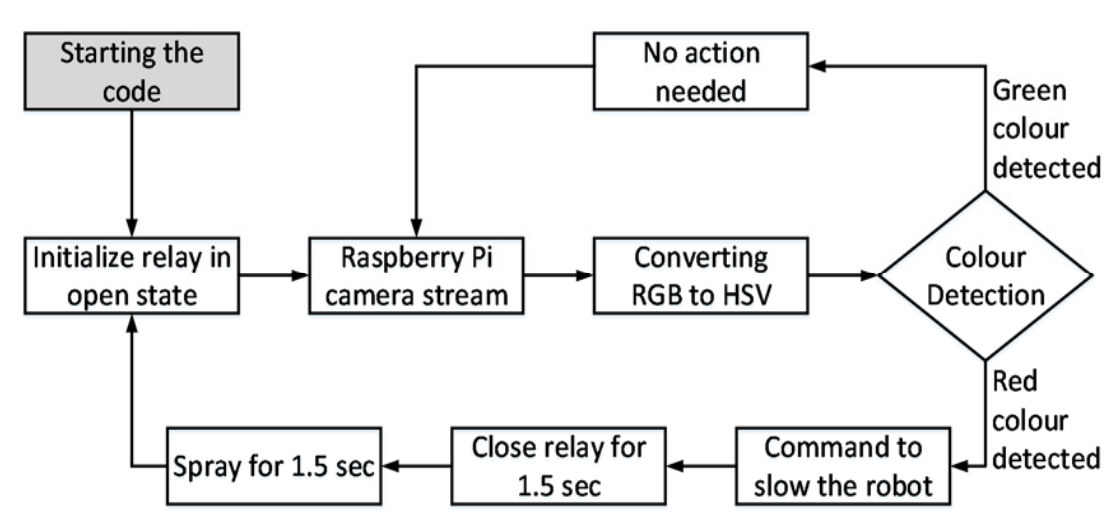

Fig. 6 Block scheme of the algorithm used for the colour detection experiment

processes. The green colour represents fresh leaves and healthy plants, while the red colour signalizes for damaged plants. The boundaries for both colours are defined in the code with small tolerance thresholds. This allows the computer vision algorithm to recognise several different shades of colours.

The detection of the red and green colours in the images requires the comparison of the values of the image pixels with the pre-defined values. If the algorithm detects the green colour or any other colour except red, no actions are invoked, and the algorithm continues to compare the other pixel values of the image. If the red colour is detected, the setAngle function is executed. With this function, a low- level signal is sent to the electrical relay switch of the robot. The duration of the signal is just 1.5 seconds and during this low-level signal, the relay is closed, which activates the motor of the sprinklers and the spraying starts. Once the 1.5 seconds have elapsed, a high-level signal is sent to the relay, which causes the spraying to stop. The duration of the process is defined in correlation to the robot speed of $12 \mathrm{~km} \cdot \mathrm{h}^{-1}$.

For our experiments, the Mission Planer software is used to make the robot navigation autonomous. This application allows the user to predefine a specific route that the robot will follow using its GPS navigation system. Once the predefined mission is uploaded to the robot, it can navigate itself along the field and can follow the planned route. During the mission, the computer vision algorithm is running constantly on the robot. Fig. 7 presents the conducted experiments - from the route planning, through the detection of the objects and then the spraying of the targeted area. Fig. 7 also presents two images with the real-time video feed from the robot and it is visible how the computer vision system of the robot has detected the green colour in the video feed (the green bench on the left), as well as the red target (red square on the right). The middle image displays the robot in the moment of spraying of the target. The experiments were conducted with a red square target with size of $20 \mathrm{~cm} \times 20 \mathrm{~cm}$. More than fifty tests were conducted with the speed of the robot ranging between 10 and $15 \mathrm{~km} \cdot \mathrm{h}^{-1}$ and the distance to the target being changed from 10 to $60 \mathrm{~cm}$. All tests were successful, and the robot was able to detect the red target and execute the spraying process.

To provide the robot with the capability to solve the SLAM problem and navigate autonomously in an unknown environment or in an environment with no or poor GPS coverage, we have developed a visual navigation solution that is based on the Intel ${ }^{\circledast}$ RealSense ${ }^{\mathrm{TM}}$ Depth Camera D435i (D435i Depth Camera,

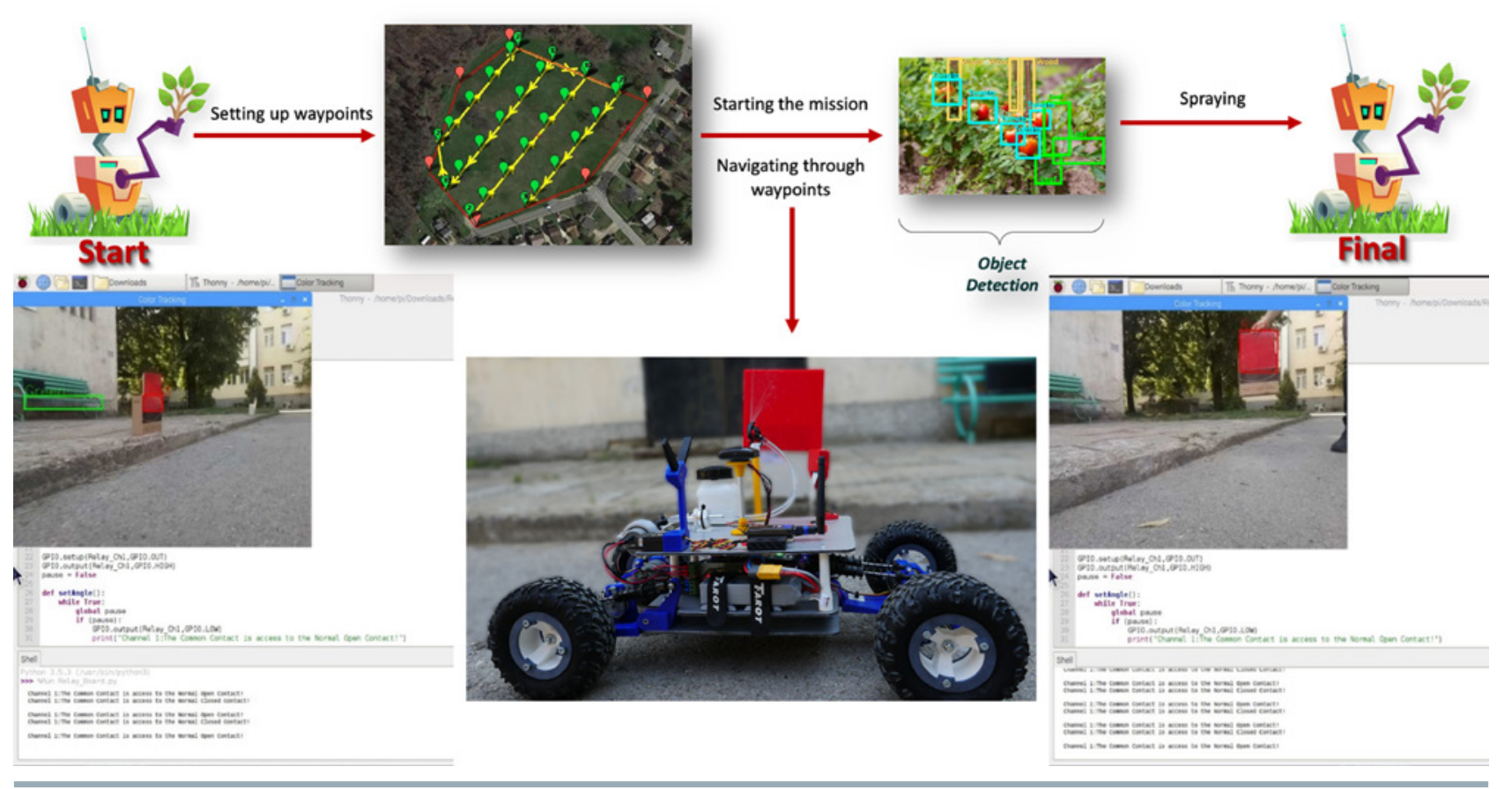

Fig. 7 Detection of green (left) and red objects (left and right) during the experiments 

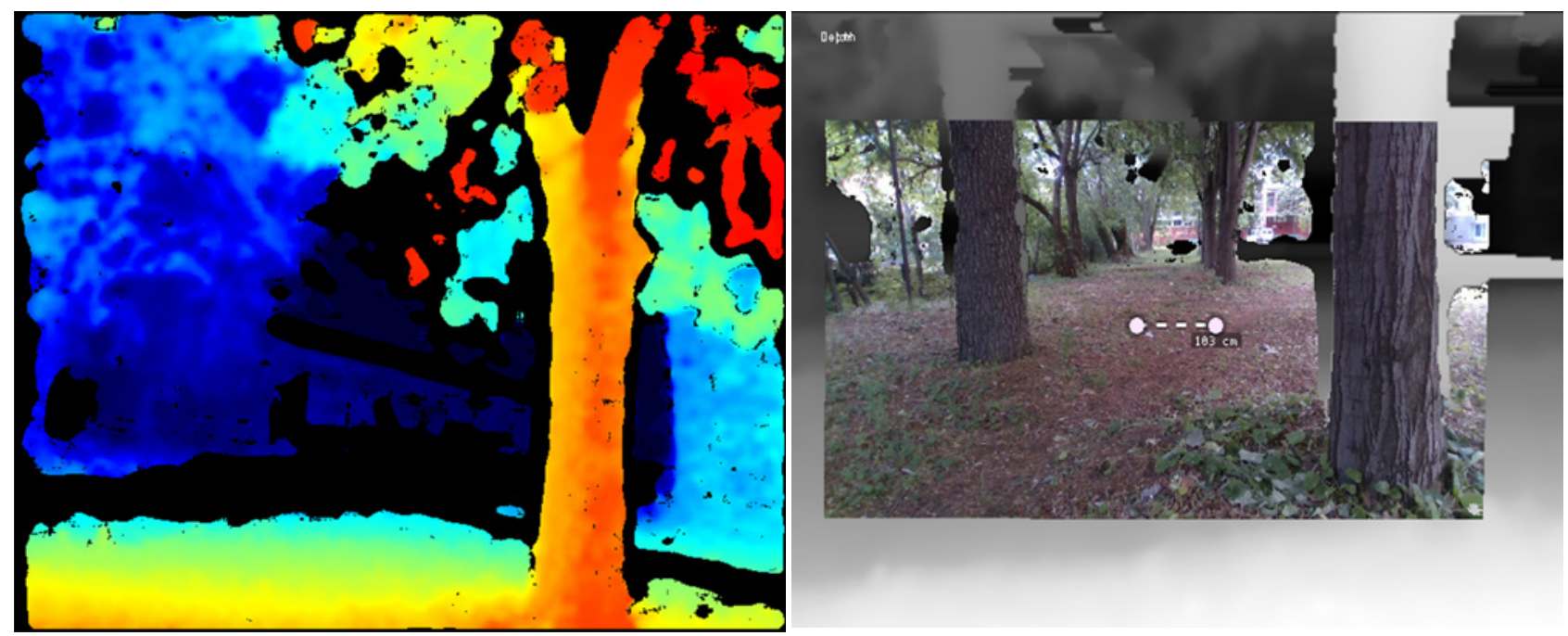

Fig. 8 Obstacle detection and avoidance using the visual navigation solution

2020) in combination with the Intel ${ }^{\circledast}$ RealSense $^{\mathrm{TM}}$ Tracking Camera T265 (T265 Tracking Camera, 2020) and the Intel ${ }^{\circledR}$ RealSense ${ }^{\mathrm{TM}}$ LiDAR Camera L515 (L515 LiDAR Camera, 2020). The use of these cameras (Fig. 8) eliminates the need for a GPS system, which otherwise is used by the robot for navigation. Being able to construct the route and not following the navigation instructions provided by the Mission planer software makes the robot fully autonomous.

Figure 8 shows how the depth camera can help the robot navigate. It shows in colour (Fig. 8 left) the

\section{The camera is facing an object 11.226 meters away}

Fig. 9 Obstacle avoidance notification

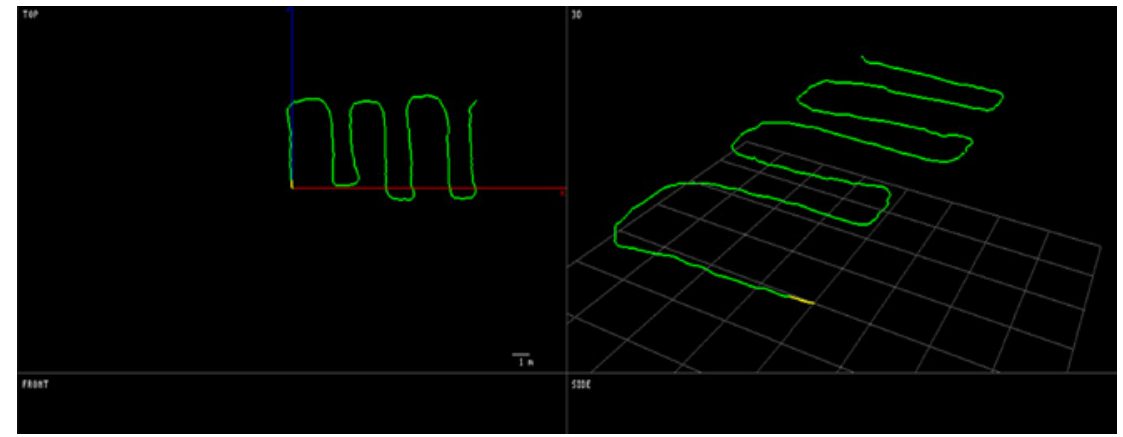

Fig. 10 The capability of the robot to track and map its own route

\section{Device Position: $0.497-0.286-1.540$ (meters) $\mathrm{d}$}

Fig. 11 Information on the current position of the robot in a relative 3D coordinate system

distance to the objects in the field of view of the robot. In this way, the robot can move towards the farthest to avoid any obstacles along

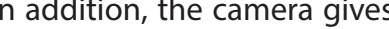
the width between the objects (Fig. 8 right). The robot is also capable of providing a feedback that contains information about the distance to the (Fig. 9). The robot is able to cope (bumps along row without damaging the plants. To avoid any obstacles, the robot will stop moving when an object is detected at

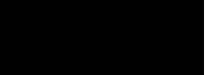


adaptive to the requirements and needs of the sector. To confirm this assumption, in the paper we have presented a prototype of a small-scale agricultural robot for precision farming. The developed prototype is in its early evaluation stage, but so far it has managed to surpass all expectations and has proven to be able to perform the basic tasks it was envisioned to do. Based on this, we can state that it is possible to develop small-scale inexpensive solutions for precision farming, based on the current information and communication technologies. The robot presented in this paper cannot fully replace the expensive commercial solutions, but can be a supplementary to them and is suitable for use both outdoors and indoors, as well as on rooftops and in urban areas. This robot also has off-road capabilities due to its high clearance and is suitable for use in farm fields. In the upcoming months, the robot will be further tested and evaluated in controlled and real environments. It will also be upgraded to perform actions that are more advanced, like weed removal, etc. The robot will also be equipped and tested to operate with manipulators, like arms, which will allow it to harvest small fruits and vegetables.

\section{Acknowledgment}

This publication is developed with the support of Project BG05M2OP001-1.001-0004 UNITe, funded under Operational Programme "Science and Education for Smart Growth", co-funded by the European Union trough the European Structural and Investment Funds.

The work presented in this paper is completed as partial fulfilment of Project 2020 - FEEA - 03 “Design and Development of a Multifunctional Robot for Implementation and Evaluation of Autonomous Navigation Algorithms", financed under the Scientific and Research Fund of the University of Ruse "Angel Kanchev".

\section{References}

123D DESIGN, 2020. Official website available at: https://www. autodesk.com/solutions/123d-apps

ABBASPOUR-GILANDEH, Y. - ABBASPOUR-GILANDEH, M. 2019. Application of computational intelligence methods for predicting soil strength. In Acta Technologica Agriculturae, vol. 22, no. 3, pp. 80-85.

AMER, G. - MUDASSIR, S. M. M. - MALIK, M. A. 2015. Design and operation of Wi-Fi agribot integrated system. In IEEE International Conference on Industrial Instrumentation and Control, pp. 207212. ISBN 978-1-4799-7165-7.

BAILEY, T. - DURRANT-WHYTE, H. 2006. Simultaneous localization and mapping (SLAM): Part II. In IEEE Robotics \& Automation Magazine, vol. 13, no. 3, pp. 108-117.

BAKKER, T. - ASSELT, K. - BONTSEMA, J. - MULLER, J. - VAN STRATEN, G. 2010. A path following algorithm for mobile robots. In Autonomous Robots, vol. 29, pp. 85-97.

BELFORTE, G. - DEBOLI, R. - GAY, P. - PICCAROLO, P. - RICAUDA AIMONINO, D. 2006. Robot design and testing for greenhouse applications. In Biosystems Engineering, vol. 95, no. 3, pp. 309-321. CHEEIN, F. A. A. - CARELLI, R. 2013. Agriculture robotics: Unmanned robotic service units in agriculture tasks. In IEEE Industrial Electronics Magazine, vol. 7, no. 3, pp. 48-58.

CLAAS 700. 2020. Official website of the Claas 700 series combiners available at: https://www.claas.co.uk/products/combines/ $\underline{\text { lexion780-740-2018 }}$
CLAAS ISARIA. 2020. Official website of the CLAAS ISARIA crops sensor available at: https://www.claas-group. com/press-corporate-communications/press-releases/ the-highly-proficient-optical-plant-sensor-from-claas/304426

COSGROVE, E. 2017. The state of play for farm robotics. Article available at: https://agfundernews.com/farm-robotics-startups. html

CVIKLOVIČ, V. - OLEJÁR, M. - HRUBÝ, D. - PALKOVÁ, Z. - LUKÁČ, O. HLAVÁČ, P. 2016. Navigation algorithm using fuzzy control method in mobile robotics. In Acta Technologica Agriculturae, vol. 19, no. 1, pp. 19-23.

D435i DEPTH CAMERA. 2020. Technical data available at: https:// www.intelrealsense.com/depth-camera-d435i/

DJI AGRAS T16. 2020. Official website available at: https://www.dji. $\mathrm{com} / \mathrm{bg} / \mathrm{t} 16$

DURRANT-WHYTE, H. - BAILEY, T. 2006. Simultaneous localization and mapping: Part I. In IEEE Robotics \& Automation Magazine, vol. 13 , no. 2, pp. 99-110.

FARMAID. 2020. Official Farmaid project page available at: https://www.hackster.io/teamato/ farmaid-plant-disease-detection-robot-55eeb1

FENDT. 2020. Official company website available at: https://www. fendt.com

HERNÁNDEZ, J. D. - BARRIENTOS, J. - DEL CERRO, J. - BARRIENTOS, A. - SANZ, A. 2013. Moisture measurement in crops using spherical robots. In Industrial Robot, vol. 40, no. 1, pp. 59-66.

HRISTOV, G. - ZAHARIEV, P. - BELOEV, I. 2016. A review of the characteristics of modern unmanned aerial vehicles. In Acta Technologica Agriculturae, vol. 19, no. 2, p. 33-38.

HUANG, J. 2017. TensorFlow object detection api, available at: https://github.com/tensorflow/models/tree/master/research/ object detection

JOHN DEERE. 2020. Official website available at: https://www.deere. com/en/agriculture

KRASTEV, G. - GEORGIEV, T. 2015. A prototype of autonomous mobile robot. In European Science and Technology - Materials of the X International Research and Practice Conference, vol. II, Munich, Germany, Vela Verlag Waldkraiburg, pp. 310-314. ISBN 978-3-941352-46-9.

L515 LiDAR CAMERA. 2020. Technical data available at: https:// www.intelrealsense.com/lidar-camera-I515/

PIERPAOLI, E. - CARLI, G. - PIGNATTI, E. - CANAVARI, M. 2013. Drivers of precision agriculture technologies adoption: A literature review. In Procedia Technology, vol. 8, pp. 61-69.

RASPBERRY 3. 2020. Official website of the Raspberry 3 Model B+ at the Raspberry Pi Foundation, available at: https://www.raspberrypi. org/products/raspberry-pi-3-model-b-plus/

T265 TRACKING CAMERA. 2020. Technical data available at: https:// www.intelrealsense.com/tracking-camera-t265/

TENSORFLOW OBJECT DETECTION ZOO. 2017. TensorFlow object detection zoo. Available at: https://github.com/tensorflow/models/ blob/master/research/object_detection/g3doc/tf1_detection zoo.md

YAGHOUBI, S. - AKBARZADEH, N. A. - BAZARGANI, S. S. BAZARGANI, S. S. - BAMIZAN, M. - ASL, M. I. 2013. Autonomous robots for agricultural tasks and farm assignment and future trends in agro robots. In International Journal of Mechanical \& Mechatronics Engineering, vol. 13, no. 3, pp. 1-6. 